\title{
Recruitment of ORC or CDC6 to DNA is sufficient to create an artificial origin of replication in mammalian cells
}

\author{
David Y. Takeda, ${ }^{1,3}$ Yoshiyuki Shibata, ${ }^{2,3}$ Jeffrey D. Parvin, ${ }^{1}$ and Anindya Dutta ${ }^{1,2,4}$ \\ ${ }^{1}$ Department of Pathology, Brigham and Women's Hospital, Harvard Medical School, Boston, Massachusetts 02115, USA; \\ ${ }^{2}$ Department of Biochemistry and Molecular Genetics, University of Virginia, Charlottesville, Virginia 22908, USA
}

Origins of replication are expected to recruit initiation proteins like origin recognition complex (ORC) and Cde6 in eukaryotes and provide a platform for unwinding DNA. Here we test whether localization of initiation proteins onto DNA is sufficient for origin function. Different components of the ORC complex and Cdc6 stimulated prereplicative complex (pre-RC) formation and replication initiation when fused to the GAL4 DNA-binding domain and recruited to plasmid DNA containing a tandem array of GAL4-binding sites. Replication occurred once per cell cycle and was inhibited by Geminin, indicating that the plasmid was properly licensed during the cell cycle. The GAL4 fusion protein recruits other polypeptides of the ORC-Cdc6 complex, and nascent strand abundance was highest near the GAL4-binding sites. Therefore, the artificial origin recapitulates many of the regulatory features of physiological origins and is valuable for studies on replication initiation in mammalian cells. We demonstrated the utility of this system by showing the functional importance of the ATPase domains of human Cde6 and Orc1 and the dispensability of the $\mathrm{N}$-terminal segments of Orc1 and Orc2 in this assay. Artificial recruitment of a eukaryotic cellular replication initiation factor to a DNA sequence can create a functional origin of replication, providing a robust genetic assay for these factors and a novel approach to generating episomal vectors for gene therapy.

[Keywords: Replication; episome; GAL4; ORC; origin; licensing; Cdc6]

Supplemental material is available at http://www.genesdev.org.

Received August 29, 2005; revised version accepted September 29, 2005.

In order to duplicate the massive eukaryotic genome in a reasonable amount of time, DNA replication begins from multiple sites located throughout the genome called replication origins (for review, see Gilbert 2001). In the budding yeast, Saccharomyces cerevisiae, replication origins are defined by specific DNA sequences $\sim 100$ base pairs $(\mathrm{bp})$ in length. Plasmids containing these sequences replicate when transformed into cells and hence are referred to as autonomously replicating sequences, or ARS. Within each ARS is an 11-bp ARS consensus sequence (ACS) that is required for binding of the six-subunit origin recognition complex (ORC). ORC remains bound to chromatin throughout the cell cycle in $S$. cerevisiae, where it catalyzes the ordered assembly of replication factors that culminate in the loading of the replicative polymerases that begin DNA synthesis (Bell and Dutta 2002; Mendez and Stillman 2003). Although ORC and many of the proteins involved in replication are con-

\footnotetext{
${ }^{3}$ These authors contributed equally to this work.

${ }^{4}$ Corresponding author.

E-MAIL ad8q@virginia.edu; FAX (434) 924-5069.

Article and publication are at http://www.genesdev.org/cgi/doi/10.1101/ gad.1369805
}

served in all eukaryotes, identification of conserved DNA sequences that define eukaryotic origins remains elusive (Gilbert 2004; Cvetic and Walter 2005). Similar plasmid transformation experiments that were successful in budding yeast have failed to identify ARS sequences in other eukaryotes. In mammalian cells, replication initiation occurs from poorly localized replication zones of 6-55 kb, although in a few cases the origin has been mapped to smaller lengths of DNA. Having welldefined origins in $S$. cerevisiae has greatly facilitated studying replication initiation. The initial discovery and characterization of ORC were done using footprinting experiments that required knowing ARS sequences (Bell and Stillman 1992). Changes that occur during initiation, such as nucleosome repositioning, could also be observed using defined ARS sequences (Lipford and Bell 2001). These and similar types of experiments are difficult to perform in other eukaryotes because of the lack of sequence specificity of origins. One means of circumventing these difficulties is to use the SV40 replication system (Waga and Stillman 1998). Replication begins in the SV40 genome at a specific 64-bp region and, in addition to cellular factors, requires a single viral proteinlarge $\mathrm{T}$ antigen. Large $\mathrm{T}$ antigen binds to the SV40 origin 
and facilitates DNA unwinding and recruitment of the replication machinery. Although the SV40 system was instrumental in identifying replication factors from mammalian cells, large $\mathrm{T}$ antigen bypasses the requirement for proteins that function prior to origin activation. Therefore, it is not suitable for studying the formation of prereplicative complexes (pre-RCs) - a major point in the cell cycle at which replication is regulated. As a result, we sought to create a new system for studying replication wherein we specify an origin in vivo by recruiting mammalian replication initiation factors to a defined DNA sequence.

Reporter assays are widely used to measure and characterize the activity of transcription factors in vivo. Typically, a transcription factor is fused to a DNA-binding domain that binds with high affinity to a specific DNA sequence. Transcription is then measured via a reporter gene placed downstream from these sequences. As a result, the transcriptional activity of a protein can be measured without requiring knowledge about what sequence it binds to in vivo. In a similar manner, if the primary function of origins is to localize initiation factors to DNA, we hypothesized that recruitment of proteins necessary for pre-RC formation to DNA will be sufficient to stimulate replication. In essence, we would create an artificial origin in vivo at a defined DNA element. We fused human replication initiation factors to the GAL4 DNA-binding domain and assessed their ability to stimulate replication of plasmids containing GAL4 DNA-binding sites. We find that recruitment of replication factors is sufficient to stimulate replication. In addition, the replication we observe is properly regulated during the cell cycle with initiation occurring in the vicinity of pre-RC assembly. We then use the origin in replication reporter assays to characterize the effect of point mutations and deletions on the activity of different replication factors.

\section{Results \\ GAL4 Orc2 and GAL4 Cdc6 stimulate replication when recruited to a plasmid}

Analogous to reporter assays for measuring the activity of transcription factors, we fused the N-terminal ends of replication initiation factors to GAL4 DNA-binding domains and assessed their ability to stimulate replication of plasmids containing GAL4 DNA-binding sites. Bacterially derived input plasmids are methylated on both strands of DpnI recognition sites and are therefore susceptible to digestion by DpnI. Since mammalian cells do not have the dam methylase enzymes, one or two cycles of replication in mammalian cells produces plasmids that are hemimethylated or unmethylated and so are, respectively, partially or totally resistant to digestion by DpnI. The appearance of DpnI-resistant DNA after transfection into mammalian cells is thus a measure of plasmid replication in the mammalian cells. A 6-kb plasmid containing five GAL4-binding sites replicated in vivo when cotransfected with plasmids expressing GAL4Orc2 or GAL4-Cde6 as determined by the detection of a DpnI-resistant band by Southern blotting (Fig. 1A, lanes
4,5). Replication was dependent on fusion to GAL4, as transfection with plasmids expressing Myc-Orc2 or Cdc6 failed to stimulate replication (Fig. 1A, lanes 6,7). Replication was also dependent on Orc2 and Cdc6, as expression of GAL4 fused to the transcription factors Sp1 or VP16 did not stimulate replication of pFR_Luc (Fig. 1A, lanes 8,9$)$. Immunoblotting or a luciferase assay for GAL4-VP16 confirmed that the absence of replication was not due to failure of the proteins to be expressed (Fig. 1E; Supplementary Fig. S1A-C). GAL4-Orc1 also stimulated replication; however, GAL4-Orc5 and GAL4-Orc6 failed to increase replication over background (Fig. 1D; Supplementary Fig. S1D), indicating that not all ORC subunits are capable of stimulating replication, although we cannot rule out negative effects due to the fusion of a GAL4 domain to these subunits.

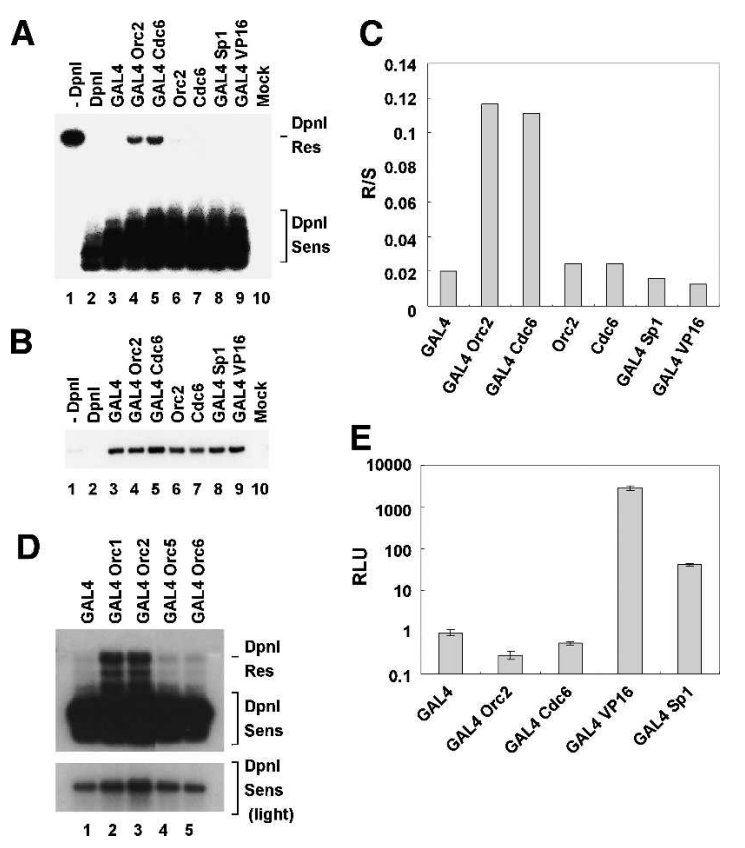

Figure 1. Replication initiation factors fused to GAL4 stimulate replication of a plasmid containing GAL4 DNA-binding sites in vivo. (A) Extrachromosomal DNA was isolated from HEK293 cells cotransfected with the indicated plasmids and pFR_Luc, which contains five GAL4-binding sites (lanes 3-10). After digestion with DpnI and NdeI $(A)$ or NdeI alone $(B)$, samples were separated by agarose gel electrophoresis, and DNA was visualized by Southern blotting using a probe to the SmaI-BstEII fragment of pFR_Luc. NdeI-digested pFR_Luc was loaded in lane 1 as a size marker for linearized plasmid. In lane 2, pFR_Luc was digested with NdeI and DpnI as a control ensuring complete digestion by DpnI. $(C)$ Replication was quantified by PhosphorImaging. (R/S) The intensity of the DpnI-resistant band in $A$ divided by the intensity of the NdeI-digested band in $B$. (D) C33a cells were transfected with the indicated plasmids and replication measured as in $A$. The bottom panel represents a lighter exposure of the top panel as a control showing equal amounts of transfected DNA. $(E)$ The transcriptional activity of the GAL4 fusions in $A$ were measured by a luciferase assay. (RLU) Firefly luciferase activity under control of GAL4binding sites was normalized to Renilla luciferase under the control of a constitutively active promoter. 
Several reports suggest that recruitment of transcription factors to chromatin alters ORC binding and replication initiation through modification of chromatin structure (Cheng et al. 1992; Aggarwal and Calvi 2004; Danis et al. 2004). However, we did not observe any stimulation of replication with two different transcription factors (Fig. 1A, lanes 8,9) despite transcription being increased 40- and 3000-fold by Sp1 and VP16, respectively (Fig. 1E). Therefore, transcription alone is not sufficient to stimulate replication in the absence of replication factors.

\section{GAL4-Orc2 and GAL4-Cdc6 promote pre-RC assembly}

We performed chromatin immunoprecipitation (ChIP) to test whether pre-RC proteins are enriched proximal to the GAL4-binding sites ("origin") relative to a site on the opposite side of the plasmid, $3 \mathrm{~kb}$ away from the GAL4binding sites ("non-origin DNA") (Fig. 2A). ChIP with anti-Orc2 showed a significant enrichment of origin DNA when assayed on cells transfected with GAL4Orc2 (Fig. 2B, left panel). In contrast, anti-Orc2 ChIP with cells transfected with Orc2 alone recovered similar levels of origin and non-origin DNA (Fig. 2B, right panel). Therefore, as expected, fusion of Orc2 to GAL4 localized Orc2 to the GAL4-binding sites. Orc1 and Orc3 also showed a significant enrichment in origin DNA over non-origin DNA, indicating that other ORC subunits are loaded at the origin by GAL4-Orc2. Antibodies to Orc6, however, failed to enrich for origin DNA. Although Orc6 is loaded at origins in S. cerevisiae, human Orc6 is not stoichiometric in the ORC complex relative to Orc1-5 (Dhar and Dutta 2000; Dhar et al. 2001a), and Orc6 has been implicated in other cellular processes outside of replication (Prasanth et al. 2002). Two different antibodies to Cdc6 (Ab-3 and H304) showed a significant enrichment of Cdc6 at origin DNA in cells transfected with GAL4-Orc2, further supporting the notion that localizing Orc2 onto DNA is sufficient to nucleate pre-RC assembly. We were unable to perform ChIP experiments with the available antibodies for Cdt1 or components of the Mcm2-7 complex. However, since overexpression of Geminin, a specific inhibitor of Cdt1, inhibits replication of the plasmid (described below), it is likely that Cdt1 is also productively recruited to the pre-RC by GAL4-Orc2.

Since Cdc6 loading is downstream of ORC loading in pre-RC assembly, we were surprised that GAL4-Cdc6 stimulated replication to the same extent as GAL4-Orc2. However, Cdc6 increases the half-life of Xenopus ORC on chromatin (Harvey and Newport 2003) and therefore may be functioning in our assay by stabilizing ORC binding to the plasmid. Indeed, Orc1, Orc2, and Orc3 were enriched at the origin in cells transfected with GAL4-Cdc6 (Fig. 2C), indicating that Cdc6 stabilizes the association of ORC subunits with DNA. To confirm that GAL4-Cdc6 requires ORC for its replicative function, we knocked down the levels of Orc1 and Orc2 using siRNA oligonucleotide duplexes. Since loss of Orc1 and Orc2 has been shown to affect cell cycle progression (Ohta et

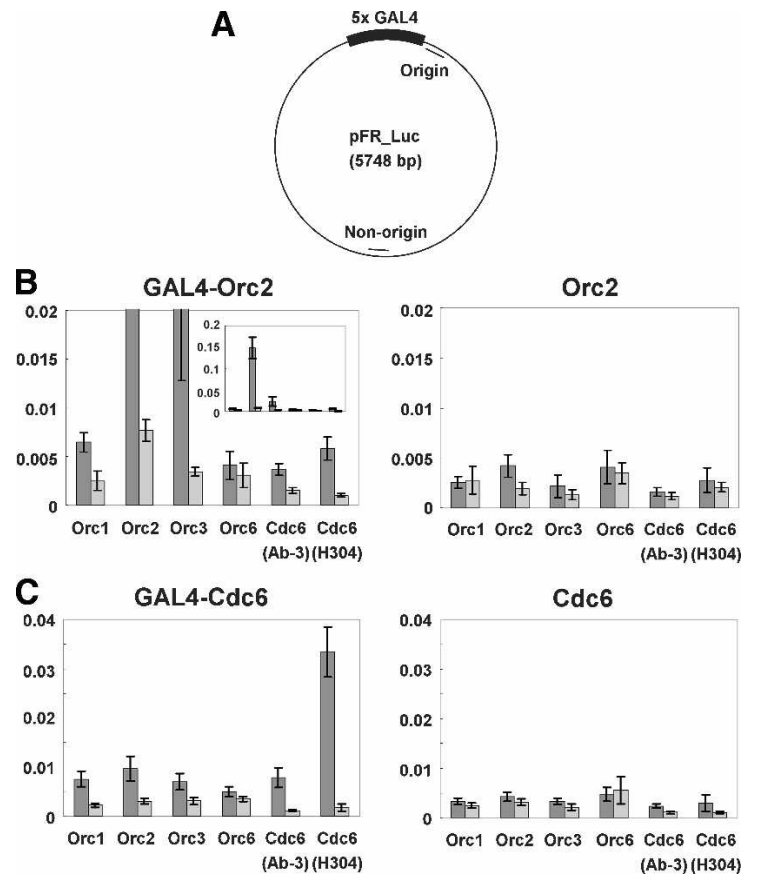

Figure 2. Pre-RC components are loaded at the artificial origin. (A) Schematic of the plasmid pFR_Luc showing the two primer sets used in ChIP assays. One set is located proximal to the GAL4-binding sites and is denoted as "Origin" DNA, while the second set is located distal to the GAL4-binding sites and is denoted as "Non-origin" DNA. (B) ChIP using chromatin from HEK293 cells transfected with plasmids expressing GAL4-Orc2 (left panel) or Orc2 (right panel). The dark bars represent amplified Origin DNA, while the light bars represent amplified nonorigin DNA. The inset shows the same plot at a different scale in order to show the results of the PCR reaction using anti-Orc2 and anti-Orc3. The $Y$-axis is the ratio of DNA in immunoprecipitate to that in input. (C) ChIP using chromatin from cells transfected with plasmids expressing GAL4-Cdc6 (left panel) or Cdc6 (right panel). The dark bars represent amplified Origin DNA, while the light bars represent amplified non-origin DNA. The $Y$-axis is the ratio of DNA in immunoprecipitate to that in input.

al. 2003; Prasanth et al. 2004; Machida et al. 2005), we titrated the amount of siRNA and used a concentration that reduced the levels of Orc1 and Orc2 (Fig. 3B) but did not alter cell cycle progression as measured by incorporation of ${ }^{3} \mathrm{H}$ thymidine (Fig. $3 \mathrm{C}$ ). Under these conditions, replication by GAL4-Cdc6 is significantly reduced compared with control cells transfected with siRNA targeting GL2 or mock-transfected cells (Fig. 3A). Therefore, GAL4-Cdc6 requires the ORC complex in order to stimulate replication.

Replication initiation properly licensed during the cell cycle

If the artificial origin was properly licensed, it should be suppressed by Geminin, a protein that binds to and inhibits the licensing factor Cdt1 (Wohlschlegel et al. 2000; Tada et al. 2001). For example, replication of EBV 
Takeda et al.

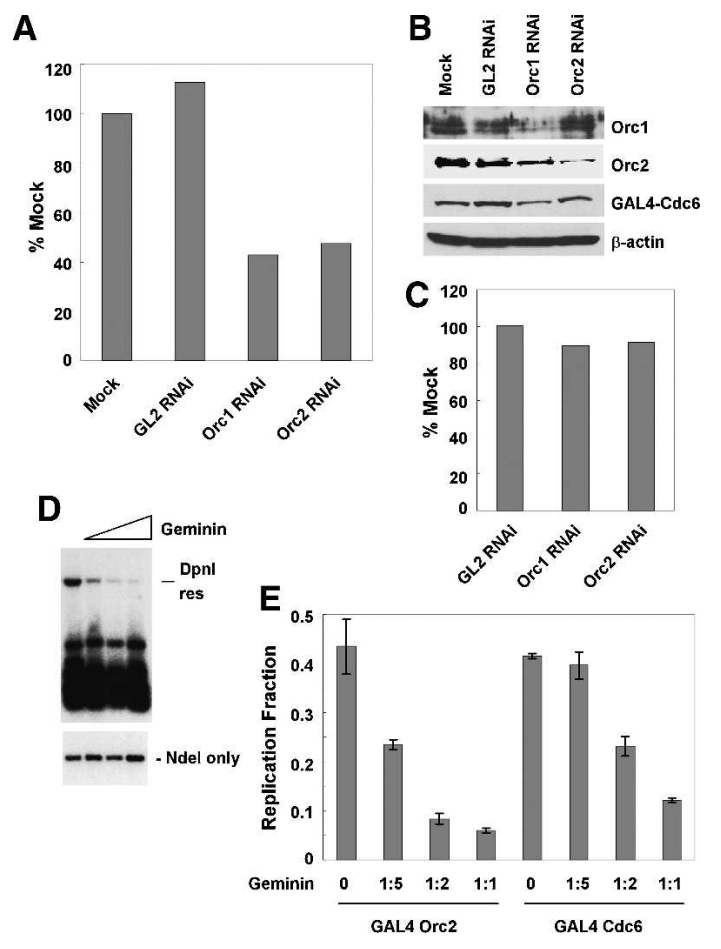

Figure 3. Replication requires Orc1 and Orc2 and is inhibited by Geminin overexpression. (A) HEK293A were cotransfected with a plasmid expressing GAL4-Cde6 and siRNA oligos to GL2, Orc1, or Orc2. Plasmid replication was measured by the quantitating the relative amount of DpnI-resistant DNA as in Figure $1(\mathrm{R} / \mathrm{S})$ and values represented as the percentage of mocktransfected samples. $(B)$ Immunoblotting of the samples in $A$ with the indicated antibodies. $(C)$ Following knock-down by siRNA of the indicated targets, cells were incubated in the presence of $\left[{ }^{3} \mathrm{H}\right]$ thymidine, and the amount of incorporated $\left[{ }^{3} \mathrm{H}\right]$ thymidine was determined by scintillation counting. Values were normalized to cell number as determined by MTT assay. (D) HEK293 cells were transfected with a plasmid expressing GAL4-Orc2 with increasing amounts of a second plasmid expressing wild-type Geminin. Replication was measured as in Figure 1. (E) HEK293 cells were transfected with plasmids expressing GAL4-Orc2 or GAL4-Cdc6 with increasing amount of Geminin expression plasmid as in $D$. The molar ratio of geminin-expressing plasmid to GAL4-Orc2 (or GAL4-Cdc6)-expressing plasmid is indicated. Extrachromosomal DNA was digested with DpnI + ExoIII or ExoIII alone, and replication was measured by quantitative real-time PCR (QPCR) using the primer set corresponding to origin DNA. The $Y$-axis is the DpnI-resistant DNA as measured by the ratio of QPCR signal from DNA digested with DpnI + ExoIII to DNA digested by ExoIII only. Identical results were obtained using the primer set that amplifies non-origin DNA (data not shown).

episomes requires the host cell's replication initiators and is inhibited by geminin (Chaudhuri et al. 2001; Dhar et al. 2001b; Schepers et al. 2001). Cotransfecting increasing amounts of a plasmid expressing wild-type Geminin with GAL4-Orc2 caused a dose-dependent inhibition of replication as detected by Southern blotting (Fig. 3D). A quantitative PCR method of measuring plasmid replication (Taylor and Morgan 2003) confirmed that Geminin decreases replication stimulated by GAL4-
Orc2 and GAL4-Cde6 in a dose-dependent manner (Fig. $3 \mathrm{E})$. Therefore, the artificial origin was regulated by Geminin.

Since the artificial origin is inhibited by Geminin, we hypothesized that the plasmid is properly licensed, thereby limiting replication to once per cell cycle. Transfected cells were blocked at G1/S and released into drugfree medium in the presence of BrdU (Fig. 4A). At 0 h, both the genomic and replicated plasmid DNA are in the light/light fractions (Fig. 4B,C). At 12 h, when most cells have progressed through $S$ phase, the genomic DNA displayed a prominent heavy/light peak and a decrease in the light/light peak. The heavy/light fractions also displayed an increase in replicated plasmid DNA, regardless of whether the replication was driven by GAL4-Orc2 (Fig. 4B) or GAL4-Cdc6 (Fig. 4C). Importantly there was no DpnI-resistant DNA in the fractions corresponding to heavy/heavy DNA, indicating that the plasmid replicates once per $S$ phase and does not go through repeated rounds of replication in the same cell cycle. At the 12-h time point, there was still a significant amount of light/ light plasmid DNA, most likely because the replication is not $100 \%$ efficient. At $36 \mathrm{~h}$ after release, about half the genomic DNA shifts to fractions corresponding to heavy/heavy DNA density, and half remains in the heavy/light fractions. Plasmid DNA is also detectable in the heavy/heavy fractions at $36 \mathrm{~h}$. The efficiency of the second round of plasmid replication is diminished relative to the first round of replication and may be partly due to dilution of the expression plasmids expressing GAL4-Orc2 and GAL4-Cdc6. The synchronous replication of plasmid and genomic DNA suggests that the artificial origin replicates once per cell cycle, similar to physiologically licensed origins.

\section{Initiation occurs preferentially near site of Orc2 and Cdco binding}

Next we determined whether replication initiation occurs preferentially near the GAL4-binding sites or is delocalized and occurs all over the plasmid (Fig. 4D). Newly replicated DNA near an origin is enriched in nascent strands: RNA-primed single-stranded DNA longer than the average Okazaki fragments (Giacca et al. 1997). Nascent-strand DNA was enriched threefold with GAL4-Orc2 and fourfold with GAL4-Cde6 at the origin DNA relative to non-origin DNA (Fig. 4E). Primer sets \#1 and $\# 2$, which are located $\sim 1.5 \mathrm{~kb}$ from the GAL4-binding sites, show an intermediate level of enrichment. The levels of enrichment and the Gaussian distribution of enrichment along the length of the plasmid are comparable to that seen in chromosomal origins at the $\beta$-globin or c-myc loci (Kamath and Leffak 2001; Aladjem et al. 2002; Liu et al. 2003; Altman and Fanning 2004) and suggest that replication initiation occurs preferentially near the GAL4-binding sites.

\section{Mapping domains of Orc1 and Cdc6}

Reporter assays are widely used to perform structurefunction studies on transcription factors. In a similar 

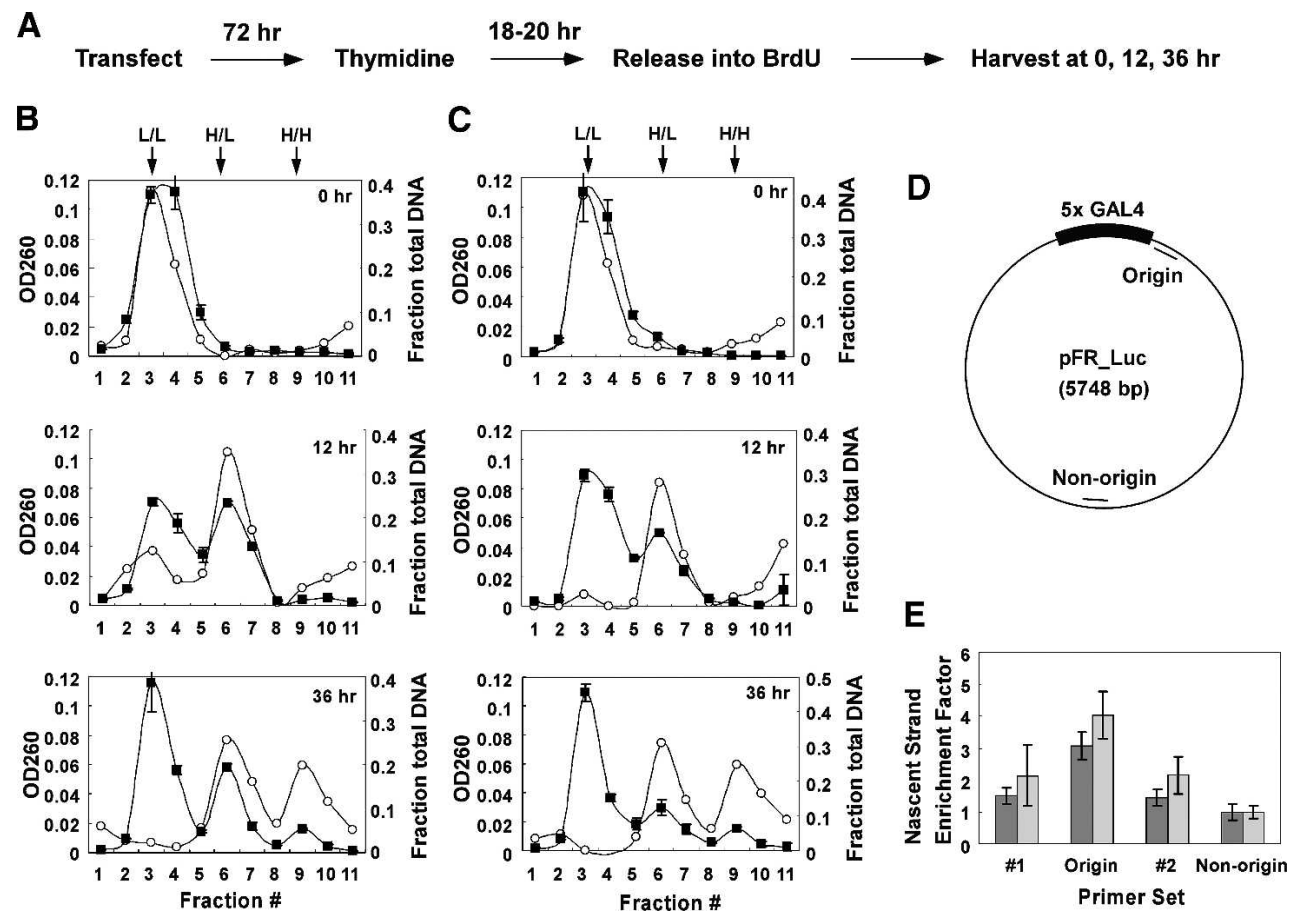

Figure 4. pFR_Luc replicates once per cell cycle with initiation localized to the GAL4-binding sites. (A) Schematic diagram of time course of experimental protocol. $(B, C)$ HEK293 cells transfected with plasmids expressing GAL4-Orc2 $(B)$ or GAL4-Cdc6 $(C)$ and pFR_luc were released from a thymidine block into medium containing BrdU and harvested 0, 12, or $36 \mathrm{~h}$ later. Genomic and extrachromosomal DNA was isolated, digested, and loaded onto a cesium chloride gradient to separate DNA of different densities. Fractions were collected, and the amount of genomic DNA in each fraction was determined by measuring the absorbance at $260 \mathrm{~nm}$ (open circles); the amount of DpnI-resistant plasmid DNA was measured by quantitative PCR (filled squares). (D) Primer pairs used to measure the nascent-strand abundance assay on pFR_luc by real-time quantitative PCR. (E) Nascent-strand DNA was purified from HEK293 cells transfected with GAL4-Orc2 (dark bars) or GAL4-Cdc6 (light bars), and DNA was quantified by quantitative PCR. The results are normalized to the amount of non-origin DNA to allow comparisons between independent experiments. Data represent the average and standard deviation of two independent experiments performed in duplicate.

fashion, we used our replication initiation assay to study the effect of point mutations on the function of Cde6 and Orc1. We made mutations in the Walker B and sensor I motifs of Cdc6 as well as the sensor I motif of Orc1. These mutations decrease replication initiation relative to the corresponding wild-type protein (Fig. 5A). Immunoblotting and immunofluorescence ruled out differences due to changes in expression or localization (Fig. $5 \mathrm{~B}, \mathrm{C})$. In contrast, mutations in the winged helix domain in the $\mathrm{C}$ terminus of Cdc6 had a minimal effect on replication initiation (Fig. 5A, lane 5). Therefore, the ATPase domain of human Orc1 or Cde6 is important for replication activity in mammalian cells, analogous to what has been found for replication in yeast or Xenopus egg extracts (Liu et al. 2000; Schepers and Diffley 2001; Frolova et al. 2002).

$N$-terminal domains of Orc1 and Orc2 are dispensable in this assay

Orc1 is partly homologous to Cdc6. Archaea and some parasitic eukaryotes have only one protein related to Orc1/Cdc6. This leads to the hypothesis that the AAA+ domain of these proteins is the functional domain and the long $\mathrm{N}$-terminal domain of Orc1 may be dispensable. We made an Orcl deletion mutant lacking the 479 amino acid residues upstream of the AAA+ domain. This mutant had the ability to stimulate replication when fused to the GAL4 DNA-binding domain (Fig. 6A).

The entire length of the Arabidopsis Orc2 homolog corresponds to the $\mathrm{C}$-terminal halves of the other known Orc2 homologs and totally lacks the $\mathrm{N}$-terminal half (Witmer et al. 2003). We have earlier reported that the $\mathrm{N}$-terminal half of human Orc2 is dispensable for interaction with Orc3 (Dhar et al. 2001a). We therefore examined whether the Orc2 $\mathrm{N}$-terminal region is required for replication by creating Orc2 mutants that lack the first 72 or 177 amino acid residues. Both Orc2 deletion mutants could support replication. Immunoblotting (Fig. $6 \mathrm{~B}$ ) and immunofluorescence data (data not shown) confirm protein expression and nuclear localization comparable to wild-type Orc2.

\section{Discussion}

These results are the first to demonstrate that the artificial recruitment of any eukaryotic replication initiation factor to a DNA sequence can create a functional 
Takeda et al.
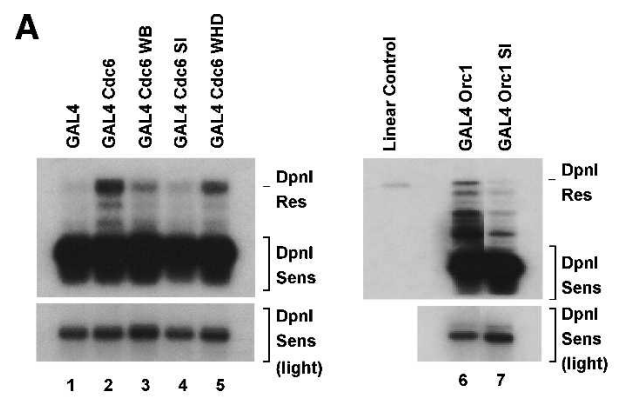

B
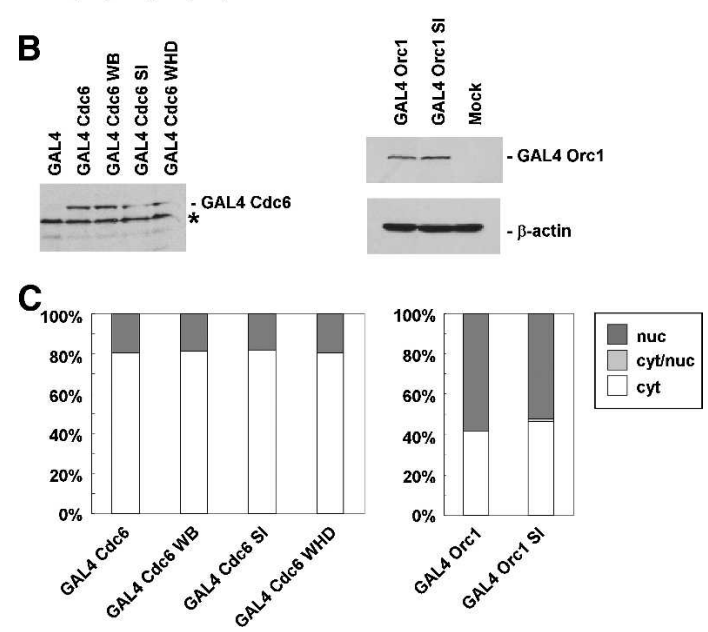

Figure 5. Activity of Cdc6 and Orc1 point mutants. (A) C33a cells were transfected with the indicated wild-type or mutant plasmid constructs of Cdc6 or Orc1, and replication of pFR_Luc was measured as in Figure 1. (WB) Walker B mutant; (S1) sensor-I mutant; (WHD) winged-helix-domain mutant. $(B)$ Immunoblotting of samples in $A$ and $B$ using anti-GAL4. Asterisks indicate a nonspecific band used as a loading control. (C) Subcellular localization of different constructs as determined by immunofluorescence using antibodies to GAL4. The percent of cells showing nuclear, cytoplasmic, and nuclear + cytoplasmic location of the GAL4 fusion protein is indicated.

origin of replication. This artificial origin provides a quick and effective means of assaying the function of mammalian initiator proteins and the regulation of the same by the licensing mechanism.

The homology between the ORC and Cdc6 polypeptides, and the presence in archaea and some parasitic eukaryotes of only one protein related to ORC/Cdc6 that forms homo-multimers, has led to the suggestion that ORC and Cdc6 work together as an AAA+ ATPase motor (Mendez and Stillman 2003). The ability of GAL4-Cdc6 to stabilize ORC subunits on DNA supports this model over the alternative model that ORC and Cde6 act sequentially. The relatively labile interactions of human ORC subunits might facilitate the assembly of the entire ORC/Cdc6 complex on the DNA after the initial binding of the GAL4 fusion protein. The binding through the GAL4 DNA-binding site still allows replication initiation. The GAL4 DNA-binding domain seems analogous to the naturally occurring AT-hook domain at the $\mathrm{N}$ terminus of Orc4 in Schizosaccharomyces pombe that has been shown to recruit ORC to AT-rich DNA segments that specify origins in S. pombe (Chuang and Kelly 1999; Lee et al. 2001). Orc4 from other species lacks this AThook domain. If the ORC/Cdc6 complex in our experiments has to directly interact with the DNA to facilitate MCM loading, then the N-terminal tethering of the complex does not appear to interfere with this interaction. Most likely, despite the N-terminal tethering, the junction between the GAL4-binding domain and the ORC/ Cdc6 complex has enough flexibility to allow the catalytic domains of ORC/Cdc6 to come into direct contact with the DNA adjoining the GAL4-binding sites. It will be interesting to test whether interpolation of rigid protein segments between GAL4 and the ORC/Cdc6 interferes with replication initiation activity.

Because vertebrate ORC lacks sequence-specific DNA-binding activity (Vashee et al. 2003; Schaarschmidt et al. 2004), it has proved difficult to demonstrate that initiation activity is physically close to ORCbinding sites. By artificially converting the ORC/Cdc6 complex into a sequence-specific DNA-binding complex, we demonstrate that nascent strands are indeed enriched near the binding site. This result suggests that the peaks of origin activity distributed over the broad initiation zones of mammalian chromosomes are likely to be proximate to ORC-binding sites (Vaughn et al. 1990; Kamath and Leffak 2001; Aladjem et al. 2002; Liu et al. 2003). It is interesting to note that even with the artificial recruitment of ORC to a specific DNA sequence, we still observed a broad peak of enrichment of nascent strands, albeit with the peak proximate to the binding site. This too is consistent with the broad peaks

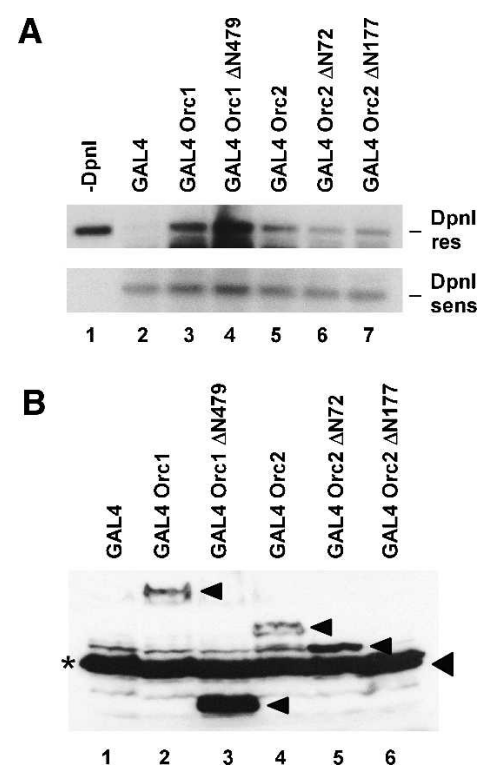

Figure 6. $\mathrm{N}$ terminus-deleted Orc1 and Orc2 can stimulate DNA replication. (A) C33a cells were transfected with wildtype or deletion constructs of Orc1 or Orc2, and replication of pFR_Luc was measured as in Figure 1. (B) Immunoblotting of samples in $A$ using anti-Gal4 showing expression of the relevant GAL4 fusion proteins. The asterisk indicates a nonspecific band. 
of initiation activity seen in zones of initiation noted in mammalian origins and suggests that if the chromatin and transcription activity permits, the initiation activity of a single pre-RC can extend over kilobases of DNA sequence.

Although they have been described now for at least a decade, detailed structure-function studies of human replication initiation factors have been lacking. This is primarily because of the lack of a convenient genetic system to assay mutant forms of the many proteins that form an active pre-RC. An elegant experiment showed that recombinant human ORC with all six subunits made and complexed in baculoviruses can substitute for the Xenopus ORC for replication in Xenopus egg extracts (Vashee et al. 2003). To assay mutants in the ORC subunits, however, would require that the mutants be expressed again in baculoviruses to be incorporated into ORC complexes that will then be purified and assayed in ORC-depleted Xenopus egg extracts. As we show with the point mutants of Orc1 and Cdc6 and the deletion mutants of Orc1 and Orc2, the functional assay of mutant forms of human ORC/Cdc6 is rendered considerably easier by the use of the artificial origin. By using this assay, we hope to systematically identify the minimal domains of ORC subunits and of Cdc6 for structural studies and to determine the importance of various structural motifs of these proteins that will become evident from structural studies. In an intriguing result, we show that the N-terminal region of human Orc1 containing an evolutionarily conserved BAH (bromo-adjoininghomology) domain that interacts with heterochromatin protein HP1, is dispensable for replication when Orc1 is targeted to the DNA by artificial fusion to the GAL4 DNA-binding domain. Of course, this does not imply that the BAH domain is dispensable for recruitment of wild-type Orc1 to origins of replication or for other functions of ORC like the establishment of heterochromatin.

Much like studies on transcription initiation, an origin-specific, cell-free replication reaction dependent on eukaryotic replication factors would be invaluable to dissect the mechanism of replication initiation. We and others have reported that the oriP origin of replication derived from the Epstein-Barr virus uses human replication initiation factors in addition to EBNA1 for replication in vivo (Chaudhuri et al. 2001; Dhar et al. 2001b; Schepers et al. 2001). It has proved difficult to extend these in vivo observations to a robust origin-specific cellfree replication reaction. The observation that GAL4Orc1, GAL4-Orc2, or GAL4-Cdc6 can support replication initiation on plasmids with GAL4 DNA-binding sites provides a new opportunity to re-establish such an origin-specific cell-free replication reaction.

The regulation of this artificial origin by the cell cycledependent licensing mechanism will open new avenues for studying disorders in this regulation. The once-percell-cycle mode of replication and inhibition by geminin suggests that the important features of the licensing mechanism are preserved in the artificial origin. Increased cdk2 activity, alteration of the geminin/Cdt1 balance, phosphorylation/ubiquitination of Cdc6/Orc1, and destruction/cytoplasmic export of Orc1 or Cdc6 have all been postulated to be important for limiting replication to once per cell cycle (for review, see Blow and Dutta 2005). Of all these factors, inhibition of Cdc2 kinase in G2 and alteration of the geminin/Cdt1 balance are the only manipulations that override the once-percell-cycle control of chromosomal DNA replication. By using mutant forms of Cdc6 or Orc1 in the GAL4 fusion protein, we can now study whether modifications of these specific proteins contribute to the once-per-cellcycle control.

Much like promoters of transcription, the efficiency of an origin of replication is likely to be influenced by cisacting elements that bind other cellular factors or influence the state of the chromatin at the origins. The artificial origin-based replication reaction described here will now allow us to manipulate these features on the plasmid and measure the effect of chromatin and other DNA-binding factors on replication initiation. An examination of the pFR-luc plasmid sequence reveals that there is a promoter in the sequence adjoining the GAL4binding sites. This promoter, however, is not required for the replication activity, as demonstrated by mutation of the TATA-box (data not shown). The 300 nucleotides immediately adjoining and upstream from the GAL4binding sites have an AT content of $66 \%$ compared with the average AT content for the entire plasmid of $55 \%$. Consistent with the higher AT content, this area has a lower helical stability and could serve as a DNA unwinding element. The average free energy required to unwind 100-bp sliding windows was calculated as $95.7 \mathrm{kcal} / \mathrm{mol}$ for the AT-rich area adjoining the GAL4-binding sites, compared with $107.36 \mathrm{kcal} / \mathrm{mol}$ for the entire plasmid (Huang and Kowalski 2003). We can now experimentally test whether these cis-acting features are important for efficient origin activity of mammalian episomes.

Episomal, nonintegrating vectors for gene therapy are considered to be less dangerous because the integration of the retroviral- or lentiviral-based vectors into the chromosomes is inherently mutagenic. Until now all episomal vectors have been derived from viral origins of replication and so require the introduction of viral proteins, raising concerns about their safety. The creation of artificial origins by fusing DNA-binding domains to human initiation factors raises the possibility of creating episomal vectors for gene therapy that are not dependent on viral proteins. Such vectors would have the advantage of episomal vectors in not being mutagenic while avoiding the problem of introducing virally derived proteins.

For the plasmid to be useful as a vector for gene therapy, we need an estimate of its replication efficiency. It is very difficult to estimate the true efficiency of replication in experiments where DpnI digestions are done directly on DNA recovered from the plates (as in Fig. 1C, where $\sim 10 \%$ of the input DNA becomes DpnI resistant), because we do not know what fraction of the plasmid put on the cultures actually enters the cells or the nuclei. We suspect that a large fraction of the DpnI-digested DNA comes from plasmids that never had a chance to replicate because they did not reach the correct compart- 
ment. In Figure 4B and C we can focus on the plasmids that have replicated at least once to produce heavy/light DNA and estimate the efficiency with which it replicates a second time to produce heavy/heavy DNA. By this estimate, it seems that once in the correct subcellular compartment, $30 \%-50 \%$ of the plasmid can replicate in a 24-h interval. The efficiency of replication can no doubt be improved by expressing the GAL4 fusion protein from the same plasmid that contains the GAL4binding sites. Alternatively, constitutive overproduction of the GAL4 fusion proteins in the cells might improve replication. Other polypeptides in the ORC/CDC6 complex or even in the steps to replication fork formation (e.g., MCMs, CDC45) may be rate limiting and so overexpression of such rate-limiting components might improve the efficiency of replication. For efficient use as a vector, the plasmid has to be maintained stably in the cells over many generations, and so another improvement would be to incorporate an element in the plasmid that promotes its attachment to chromosomes or the mitotic spindle to ensure proper segregation to the daughter cells. Addition of a centromere could also be useful, but might be difficult due to the large size of mammalian centromeres.

In conclusion, we have created a functional origin of replication in vivo by recruiting replication factors to a specific DNA sequence. By defining the site of replication initiation, we are now in a position to perform controlled studies on the function of initiators at origins in mammalian cells. Importantly, initiation from this origin is properly regulated during the cell cycle and should facilitate studies on replication licensing and the regulation of replication initiation during the cell cycle. The results also have practical implications for the development of episomal vectors for gene therapy.

\section{Materials and methods}

\section{Plasmids}

Plasmids expressing GAL4-Orc1, GAL4-Orc2, GAL4-Orc5, GAL4-Orc6, and GAL4-Cdc6 were constructed by amplifying the appropriate open reading frame by PCR using primers engineered with restriction sites and cloned into the $\mathrm{pM}$ vector (Clontech) using the SmaI and MluI sites for Orc2 and the BamHI and MluI sites for Orc1, Orc5, Orc6, and Cdc6. The reporter plasmid pFR_Luc is commercially available from Stratagene. The expression vector for Myc-tagged Orc2 was made by cloning the ORF of Orc2 into the AgeI and NheI sites of pA3M. We used pCDNA3_Cdc6 for expression of untagged Cdc6 (Saha et al. 1998). The expression vector for Geminin (pEBG_Geminin) has been described elsewhere (Wohlschlegel et al. 2002).

\section{Cell culture, transfections, RNA interference (RNAi)}

Human Embryonic Kidney 293A cells were grown as adherent monolayers in Dulbecco's modified Eagle's medium supplemented with $10 \%$ donor calf serum. For synchronization at G1/ $\mathrm{S}$, cells were incubated for $20 \mathrm{~h}$ in the presence of $2 \mathrm{mM}$ thymidine. Cells were transfected using Lipofectamine 2000 (Invitrogen) according to manufacturer's instructions and harvested 3 $\mathrm{d}$ later. For RNAi experiments, RNA duplexes were synthesized
(Invitrogen) targeting the sequence 5'-AAGGUCCCGGCU CAAGCAUGU-3' in Orc1 and 5'-AAGAAGGAGCGAGCG CAGCUU-3' in Orc2. Cervical cancer C33a cells were transfected with $25 \mathrm{nM}$ siRNA duplex in $10-\mathrm{cm}$ plates at $12 \%$ confluency using oligofectamine (Invitrogen). Transfections were repeated once after $24 \mathrm{~h}$.

\section{Transient transfection replication assay}

Extrachromosomal DNA was enriched by the method of Hirt (1967). Cells were lysed with TE containing 1\% SDS followed by the addition of $5 \mathrm{M} \mathrm{NaCl}$ to a final concentration of $1 \mathrm{M}$ $\mathrm{NaCl}$. After incubation overnight at $4^{\circ} \mathrm{C}$, lysates were spun at $15,000 \mathrm{~g}$ for $30 \mathrm{~min}$ and treated with proteinase $\mathrm{K}(100 \mu \mathrm{g} / \mathrm{mL})$ for $1 \mathrm{~h}$ at $37^{\circ} \mathrm{C}$. DNA was isolated by phenol-chloroform extraction followed by ethanol precipitation, and the DNA pellet was resuspended in $10 \mathrm{mM}$ (Tris at $\mathrm{pH} 8.0$ ) buffer. For Southern blotting, DNA was incubated overnight at $37^{\circ} \mathrm{C}$ with NdeI and DpnI or with NdeI alone (NEB). Digested DNA was separated on a $1 \%$ agarose gel and blotted according to standard protocols for Southern blotting. The probe we used corresponded to the BstEII-SmaI fragment of pFR_Luc. Band intensity was quantified using PhosphorImaging (Molecular Dynamics) and normalized by dividing the value for the DpnI-resistant band by the value for the NdeI-digested band. For measuring replication by real-time PCR, isolated DNA was digested with DpnI and Exonuclease III (NEB) to reduce contaminating DNA as described previously (Taylor and Morgan 2003).

\section{Quantitative real-time PCR}

Quantitative PCR reactions were performed using the Bio-Rad iQSybr platform with iQSybr PCR mix (Bio-Rad) in a total volume of $50 \mu \mathrm{L}$. We used four 10-fold dilutions of pFR_Luc to generate a standard curve, and all of the primer pairs used in our measurements had amplification efficiencies $>97 \%$. The amount of DNA in samples was extrapolated from the standard curves. The primer sets used are as follows: Origin DNA: 5'CTTGGCATTCCGGTACTGTT-3' and 5'-CCTTATGCAGT TGCTCTCCA-3'; Non-origin DNA: 5'-CCAGGAAGCTCCT CTGTGT-3' and 5'-GGATGGGCAGCCTATGATT-3'; Set \#1: 5'-TGTTTGTGGACGAAGTACCG- ${ }^{\prime}$ ' and $5^{\prime}$-CCCTTCTTG GCCTTTATGAG-3'; Set \#2: 5'-TTCCTGCGTTATCCCTG ATT-3' and 5'-CTTCCTCGCTCACTGACTTGC-3'.

\section{Immunoblotting and antibodies}

Immunoblotting was performed according to standard procedures using the following antibodies: GAL4 DBD RK5C1 (Santa Cruz), Cdc6 Ab-3 (Calbiochem), Cdc6 H304 (Santa Cruz), and Orc1, Orc2, Orc 3, Orc 5, and Orc6 (Dhar and Dutta 2000).

\section{ChIP}

Cells were fixed with $1 \%$ formaldehyde for $10 \mathrm{~min}$, quenched with 2.5 M glycine, and scraped into a 50-mL conical tube. After washing twice with cold PBS, cells were resuspended in $10 \mathrm{mM}$ Tris (pH 8.0), $200 \mathrm{mM} \mathrm{NaCl}, 1 \mathrm{mM}$ EDTA, 0.5 mM EGTA, and $0.25 \%$ Triton, and further lysed by douncing. After centrifugation, pellets were resuspended in $10 \mathrm{mM}$ Tris $(\mathrm{pH} 8.0), 1 \mathrm{mM}$ EDTA, $0.5 \mathrm{mM}$ EGTA, and 1\% Sarkosyl, and sonicated using a Branson 450. Lysates were spun at $15,000 \mathrm{rpm}$ for $10 \mathrm{~min}$ to remove debris, and the supernatant was loaded onto a cesium chloride gradient containing $7.381 \mathrm{~g}$ of $\mathrm{CsCl}$ in $13 \mathrm{~mL}$ of $\mathrm{TE}$ with $1 \%$ Sarkosyl and spun at $50,000 \mathrm{rpm}$ for $24 \mathrm{~h}$ at $20^{\circ} \mathrm{C}$ in an 
NVT65 rotor. Fractions of $1 \mathrm{~mL}$ were collected, and those corresponding to a density of $1.4 \mathrm{~g} / \mathrm{cm}^{3}$ were pooled and dialyzed twice (Spectra/Por Membrane M.W.C.O. 3500) against $20 \mathrm{mM}$ Tris (pH 8), $150 \mathrm{mM} \mathrm{NaCl}, 1 \mathrm{mM}$ EDTA, $0.5 \mathrm{mM}$ EGTA, 1\% Triton, and $0.01 \%$ SDS at $4^{\circ} \mathrm{C}$. Appropriate antibodies were added to the samples and incubated overnight at $4^{\circ} \mathrm{C}$. Protein G-Sepharose (Pharmacia) was added and incubated for $1 \mathrm{~h}$ at $4^{\circ} \mathrm{C}$. Immunoprecipitates were then washed three times for 5 min each with buffer I ( $10 \mathrm{mM}$ Tris at $\mathrm{pH} 8.0,1 \mathrm{mM}$ EDTA, 150 $\mathrm{mM} \mathrm{NaCl})$, buffer II (10 mM Tris at $\mathrm{pH} 8.0,1 \mathrm{mM}$ EDTA, 500 $\mathrm{mM} \mathrm{NaCl})$, buffer III (10 mM Tris at $\mathrm{pH}$ 8.0, 1 mM EDTA, 250 $\mathrm{mM} \mathrm{LiCl)}$, and TE. DNA was eluted with $20 \mathrm{mM}$ Tris (pH 6.8), $2 \%$ SDS for $15 \mathrm{~min}$ at room temperature, and cross-links were reversed by incubating overnight at $65^{\circ} \mathrm{C}$ in the presence of Proteinase K $(100 \mu \mathrm{g} / \mathrm{mL})$. After phenol-chloroform extraction and ethanol precipitation, the DNA was resuspended in TE and quantified by real-time PCR. Results were normalized by dividing by input DNA (before addition of antibody).

\section{Transcription reporter assays}

Transcription was measured by the dual luciferase reporter assay (Promega) according to the manufacturer's instructions.

\section{Density gradient substitution experiments}

Two days after transfection, HEK293 cells were synchronized at the G1/S transition by incubating with $2 \mathrm{mM}$ thymidine (Sigma) for $18 \mathrm{~h}$. Cells were washed with drug-free medium and released into medium containing $30 \mu \mathrm{M}$ BrdU (Sigma). Cells were harvested at the appropriate time points and divided evenly into two samples. Genomic DNA was harvested from one sample according to standard protocols, and extrachromosomal DNA was isolated from the second sample by Hirt extraction. Genomic DNA was digested with SmaI, ApaI, and RNase, and plasmid DNA was digested with DpnI and ExoIII overnight. The samples were then loaded onto a cesium chloride gradient with a refractive index of 1.4038 corresponding to a density of $1.75 \mathrm{~g} / \mathrm{cm}^{3}$ in a total volume of $13.5 \mathrm{~mL}$. After ultracentrifugation at $50,000 \mathrm{rpm}$ for $20 \mathrm{~h}$ at $20^{\circ} \mathrm{C}$ in a VTi65 rotor, $1-\mathrm{mL}$ fractions were collected and dialyzed twice against TE followed by ethanol precipitation. Genomic DNA was measured by absorbance at $260 \mathrm{~nm}$, and plasmid DNA was quantified by real-time PCR.

\section{Purification of nascent-strand DNA}

Nascent-strand DNA was purified essentially as described elsewhere (Gerbi and Bielinsky 1997). Genomic DNA from $1-2 \times 10^{8}$ cells was isolated according to standard procedures and loaded onto a BND cellulose column, which isolates singlestranded DNA. The column was washed with 10 volumes of NET buffer ( $1 \mathrm{M} \mathrm{NaCl}, 1 \mathrm{mM}$ EDTA, $10 \mathrm{mM}$ at $\mathrm{pH} 8.0)$ and eluted with 1.5 volumes of NET containing $1.8 \%$ caffeine (Sigma). DNA was concentrated by ethanol precipitation and treated with T4 polynucleotide kinase for $1 \mathrm{~h}$ at $37^{\circ} \mathrm{C}$, followed by overnight treatment with $\lambda$ exonuclease at $37^{\circ} \mathrm{C}$. After phenol-chloroform extraction and ethanol precipitation, the DNA was separated on a $1 \%$ alkaline agarose gel run in $50 \mathrm{mM}$ $\mathrm{NaOH}, 1 \mathrm{mM}$ EDTA with 1-kb and 500-bp DNA ladders (NEB). Fractions corresponding to $0.5-1.5 \mathrm{~kb}$ were excised from the gel, and DNA was purified using the QIAGEN QIAEXII gel extraction kit. Nascent-strand DNA was quantified by real-time PCR. Results were normalized by dividing by input DNA, which represented an aliquot taken prior to electrophoresis.

\section{Immunofluorescence}

C33a cells seeded on the coverslips were fixed with $2 \%$ paraformaldehyde in PBS for $15 \mathrm{~min}$ at room temperature, permeabilized with $0.2 \%$ Triton X-100 in PBS, and blocked for 30 min with $1 \%$ goat serum in PBS. Cells were incubated with antiGAL4 for $1 \mathrm{~h}$ at room temperature, washed with $1 \%$ goat serum in PBS, and stained with FITC-conjugated anti-rabbit IgG (Dako). DNA was counterstained with mounting solution containing DAPI (Vector Lab., VECTASHIELD) and analyzed by fluorescence microscopy.

\section{Tritiated thymidine incorporation assay}

Cells were plated in $24-w e l l$ plates at a density of $2.5 \times 10^{4}$ cells/ well. The following day, cells were incubated with medium containing $5 \mu \mathrm{Ci} / \mathrm{mL}\left[{ }^{3} \mathrm{H}\right]$ thymidine for $2 \mathrm{~h}$. Cells from each well were collected and precipitated using 5\% TCA. Pellets were dissolved in $0.5 \mathrm{M} \mathrm{NaOH} / 0.5 \%$ SDS, and radioactivity was measured by liquid scintillation counting. The data were normalized by cell number as determined by MTT assay (Promega).

\section{Acknowledgments}

We thank Ankit Malhotra for the AT content and $\Delta \mathrm{G}$ calculations of pFR_luc. This work was supported by grants from the NIH to A.D. (CA60499, CA89406) and J.D.P. (GM053504) and a predoctoral fellowship from Howard Hughes Medical Institute to D.Y.T.

\section{References}

Aggarwal, B.D. and Calvi, B.R. 2004. Chromatin regulates origin activity in Drosophila follicle cells. Nature 430: 372-376.

Aladjem, M.I., Rodewald, L.W., Lin, C.M., Bowman, S., Cimbora, D.M., Brody, L.L., Epner, E.M., Groudine, M., and Wahl, G.M. 2002. Replication initiation patterns in the $\beta$-globin loci of totipotent and differentiated murine cells: Evidence for multiple initiation regions. Mol. Cell. Biol. 22: 442-452.

Altman, A.L. and Fanning, E. 2004. Defined sequence modules and an architectural element cooperate to promote initiation at an ectopic mammalian chromosomal replication origin. Mol. Cell. Biol. 24: 4138-4150.

Bell, S.P. and Dutta, A. 2002. DNA replication in eukaryotic cells. Annu. Rev. Biochem. 71: 333-374.

Bell, S.P. and Stillman, B. 1992. ATP-dependent recognition of eukaryotic origins of DNA replication by a multiprotein complex. Nature 357: 128-134.

Blow, J.J. and Dutta, A. 2005. Preventing re-replication of chromosomal DNA. Nat. Rev. Mol. Cell. Biol. 6: 476-486.

Chaudhuri, B., Xu, H., Todorov, I., Dutta, A., and Yates, J.L. 2001. Human DNA replication initiation factors, ORC and MCM, associate with oriP of Epstein-Barr virus. Proc. Natl. Acad. Sci. 98: 10085-10089.

Cheng, L.Z., Workman, J.L., Kingston, R.E., and Kelly, T.J. 1992. Regulation of DNA replication in vitro by the transcriptional activation domain of GAL4-VP16. Proc. Nat1. Acad. Sci. 89: 589-593.

Chuang, R.Y. and Kelly, T.J. 1999. The fission yeast homologue of Orc4p binds to replication origin DNA via multiple AThooks. Proc. Natl. Acad. Sci. 96: 2656-2661.

Cvetic, C. and Walter, J.C. 2005. Eukaryotic origins of DNA replication: Could you please be more specific? Semin. Cell Dev. Biol. 16: 343-353. 
Danis, E., Brodolin, K., Menut, S., Maiorano, D., Girard-Reydet, C., and Mechali, M. 2004. Specification of a DNA replication origin by a transcription complex. Nat. Cell Biol. 6: 721-730.

Dhar, S.K. and Dutta, A. 2000. Identification and characterization of the human ORC6 homolog. I. Biol. Chem. 275: 34983-34988.

Dhar, S.K., Delmolino, L., and Dutta, A. 2001a. Architecture of the human origin recognition complex. J. Biol. Chem. 276: 29067-29071.

Dhar, S.K., Yoshida, K., Machida, Y., Khaira, P., Chaudhuri, B., Wohlschlegel, J.A., Leffak, M., Yates, J., and Dutta, A. 2001b. Replication from oriP of Epstein-Barr virus requires human ORC and is inhibited by geminin. Cell 106: 287-296.

Frolova, N.S., Schek, N., Tikhmyanova, N., and Coleman, T.R. 2002. Xenopus Cdc6 performs separate functions in initiating DNA replication. Mol. Biol. Cell 13: 1298-1312.

Gerbi, S.A. and Bielinsky, A.K. 1997. Replication initiation point mapping. Methods 13: 271-280.

Giacca, M., Pelizon, C., and Falaschi, A. 1997. Mapping replication origins by quantifying relative abundance of nascent DNA strands using competitive polymerase chain reaction. Methods 13: 301-312.

Gilbert, D.M. 2001. Making sense of eukaryotic DNA replication origins. Science 294: 96-100.

- 2004. In search of the holy replicator. Nat. Rev. Mol. Cell. Biol. 5: 848-855.

Harvey, K.J. and Newport, J. 2003. Metazoan origin selection: Origin recognition complex chromatin binding is regulated by CDC6 recruitment and ATP hydrolysis. J. Biol. Chem. 278: 48524-48528.

Hirt, B. 1967. Selective extraction of polyoma DNA from infected mouse cell cultures. J. Mol. Biol. 26: 365-369.

Huang, Y. and Kowalski, D. 2003. Web-thermodyn: Sequence analysis software for profiling DNA helical stability. Nucleic Acids Res. 31: 3819-3821.

Kamath, S. and Leffak, M. 2001. Multiple sites of replication initiation in the human $\beta$-globin gene locus. Nucleic Acids Res. 29: 809-817.

Lee, J.K., Moon, K.Y., Jiang, Y., and Hurwitz, J. 2001. The Schizosaccharomyces pombe origin recognition complex interacts with multiple AT-rich regions of the replication origin DNA by means of the AT-hook domains of the spOrc4 protein. Proc. Natl. Acad. Sci. 98: 13589-13594.

Lipford, J.R. and Bell, S.P. 2001. Nucleosomes positioned by ORC facilitate the initiation of DNA replication. Mol. Cell 7: 21-30.

Liu, J., Smith, C.L., DeRyckere, D., DeAngelis, K., Martin, G.S., and Berger, J.M. 2000. Structure and function of Cdc6/ Cdc18: Implications for origin recognition and checkpoint control. Mol. Cell 6: 637-648.

Liu, G., Malott, M., and Leffak, M. 2003. Multiple functional elements comprise a mammalian chromosomal replicator. Mol. Cell. Biol. 23: 1832-1842.

Machida, Y.J., Teer, J.K., and Dutta, A. 2005. Acute reduction of an origin recognition complex (ORC) subunit in human cells reveals a requirement of ORC for Cdk2 activation. J. Biol. Chem. 280: 27624-27630.

Mendez, J. and Stillman, B. 2003. Perpetuating the double helix: Molecular machines at eukaryotic DNA replication origins. Bioessays 25: 1158-1167.

Ohta, S., Tatsumi, Y., Fujita, M., Tsurimoto, T., and Obuse, C. 2003. The ORC1 cycle in human cells: II. Dynamic changes in the human ORC complex during the cell cycle. J. Biol. Chem. 278: 41535-41540.

Prasanth, S.G., Prasanth, K.V., and Stillman, B. 2002. Orc6 involved in DNA replication, chromosome segregation, and cytokinesis. Science 297: 1026-1031.

Prasanth, S.G., Prasanth, K.V., Siddiqui, K., Spector, D.L., and Stillman, B. 2004. Human Orc2 localizes to centrosomes, centromeres and heterochromatin during chromosome inheritance. EMBO J. 23: 2651-2663.

Saha, P., Chen, J., Thome, K.C., Lawlis, S.J., Hou, Z.H., Hendricks, M., Parvin, J.D., and Dutta, A. 1998. Human CDC6/ Cdc18 associates with Orc1 and cyclin-cdk and is selectively eliminated from the nucleus at the onset of $S$ phase. Mol. Cell. Biol. 18: 2758-2767.

Schaarschmidt, D., Baltin, J., Stehle, I.M., Lipps, H.J., and Knippers, R. 2004. An episomal mammalian replicon: Sequenceindependent binding of the origin recognition complex. EMBO J. 23: 191-201.

Schepers, A. and Diffley, J.F. 2001. Mutational analysis of conserved sequence motifs in the budding yeast Cdc6 protein. J. Mol. Biol. 308: 597-608.

Schepers, A., Ritzi, M., Bousset, K., Kremmer, E., Yates, J.L., Harwood, J., Diffley, J.F., and Hammerschmidt, W. 2001. Human origin recognition complex binds to the region of the latent origin of DNA replication of Epstein-Barr virus. EMBO J. 20: 4588-4602.

Tada, S., Li, A., Maiorano, D., Mechali, M., and Blow, J.J. 2001. Repression of origin assembly in metaphase depends on inhibition of RLF-B/Cdt1 by geminin. Nat. Cell Biol. 3: 107113.

Taylor, E.R. and Morgan, I.M. 2003. A novel technique with enhanced detection and quantitation of HPV-16 E1- and E2mediated DNA replication. Virology 315: 103-109.

Vashee, S., Cvetic, C., Lu, W., Simancek, P., Kelly, T.J., and Walter, J.C. 2003. Sequence-independent DNA binding and replication initiation by the human origin recognition complex. Genes \& Dev. 17: 1894-1908.

Vaughn, J.P., Dijkwel, P.A., and Hamlin, J.L. 1990. Replication initiates in a broad zone in the amplified $\mathrm{CHO}$ dihydrofolate reductase domain. Cell 61: 1075-1087.

Waga, S. and Stillman, B. 1998. The DNA replication fork in eukaryotic cells. Annu. Rev. Biochem. 67: 721-751.

Witmer, X., Alvarez-Venegas, R., San-Miguel, P., Danilevskaya, O., and Avramova, Z. 2003. Putative subunits of the maize origin of replication recognition complex ZmORC1ZmORC5. Nucleic Acids Res. 31: 619-628.

Wohlschlegel, J.A., Dwyer, B.T., Dhar, S.K., Cvetic, C., Walter, J.C., and Dutta, A. 2000. Inhibition of eukaryotic DNA replication by geminin binding to Cdt1. Science 290: 2309-2312.

Wohlschlegel, J.A., Kutok, J.L., Weng, A.P., and Dutta, A. 2002. Expression of geminin as a marker of cell proliferation in normal tissues and malignancies. Am. J. Pathol. 161: 267273. 


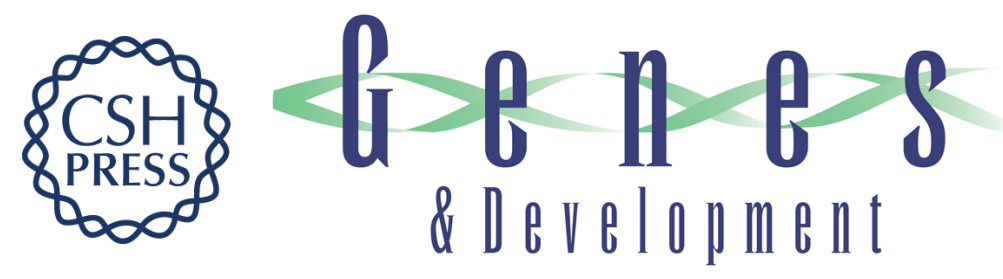

\section{Recruitment of ORC or CDC6 to DNA is sufficient to create an artificial origin of replication in mammalian cells}

David Y. Takeda, Yoshiyuki Shibata, Jeffrey D. Parvin, et al.

Genes Dev. 2005, 19:

Access the most recent version at doi:10.1101/gad.1369805

Supplemental
Material http://genesdev.cshlp.org/content/suppl/2005/11/15/19.23.2827.DC1

References This article cites 44 articles, 21 of which can be accessed free at:

http://genesdev.cshlp.org/content/19/23/2827.full.html\#ref-list-1

License

Email Alerting Receive free email alerts when new articles cite this article - sign up in the box at the top

Service right corner of the article or click here.

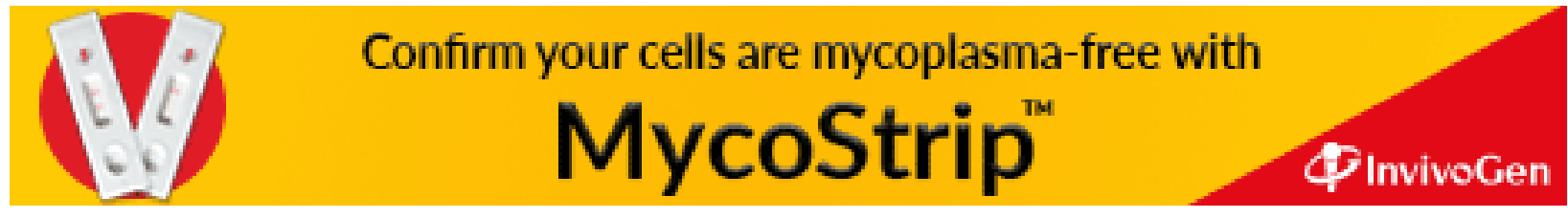

